This is a self-archived - parallel published version of this article in the publication archive of the University of Vaasa. It might differ from the original.

\title{
CryptoKitties and the new ludic economy : how blockchain introduces value, ownership, and scarcity in digital gaming
}

Author(s): Serada, Alesja; Sihvonen, Tanja; Harviainen, J. Tuomas

Title: CryptoKitties and the new ludic economy : how blockchain introduces value, ownership, and scarcity in digital gaming

Year: $\quad 2020$

Version: Accepted manuscript

Copyright (C)2020 the author(s), licensee SAGE Publications. The article is protected by copyright and reuse is restricted to noncommercial and no derivative uses. Users may also download and save a local copy of an article accessed in an institutional repository for the user's personal reference.

\section{Please cite the original version:}

Serada, A., Sihvonen, T., \& Harviainen, J.T. (2020). CryptoKitties and the new ludic economy : how blockchain introduces value, ownership, and scarcity in digital gaming. Games and culture. https://doi.org/10.1177/1555412019898305 


\title{
CryptoKitties and the New Ludic Economy: \\ How blockchain introduces value, ownership, and scarcity in digital gaming
}

\begin{abstract}
This article analyzes specific characteristics of value created through digital scarcity and blockchain-proven ownership in cryptogames. Our object of study is CryptoKitties, the first instance of a blockchain-based game that has garnered media recognition and financial interest. The objective of this article is to demonstrate the limits of scarcity in value construction for owners of CryptoKitties tokens, manifested as breedable virtual cats. Our work extends the trends set out by earlier cryptocurrency studies from the perspective of cultural studies. For the purpose of this article, we rely on open blockchain analytics such as DappRadar and Etherscan, as well as playercreated analytics, backed by a one-year-long participant observation period in the said game for research material. Combining theoretical cryptocurrency and Bitcoin studies, open data analysis, and virtual ethnography enables a grounded discussion on blockchain-based game design and play.
\end{abstract}

\section{Introduction}

Using games to grasp the functionalities of cryptocurrencies and the blockchain technologies that make them possible seems like an obvious choice. Cryptocurrencies are monetary instruments built upon the technologies collectively known as blockchains that are supposedly almost unalterable records of (usually distributed) online transactions (Campbell-Verduyn, 2018). They were first introduced by the individual or group using the name Satoshi Nakamoto in 2008 (Nakamoto, 2008), and demonstrated in action by the implementation of Bitcoin, the best-known cryptocurrency, by the same party in the following year. Cryptogames, in turn, are games that use either blockchain technology, cryptocurrencies as payments, or (typically) both. Cryptocurrencies almost have a "genetic" relationship with games: for instance, Mt. Gox, the most important Bitcoin exchange, originally started as a website dedicated to the collectible card game Magic the Gathering (Kavanagh et al., 2019). The Bitcoin economy has been interpreted as a game of sorts by many authors (e.g. Hütten \& Thiemann, 2018). Kavanagh et al. (2019) go as far as connecting the rise of Bitcoin to the birth of ludology (or game studies) by a mysterious 'Zeitgeist'.

Even though the ludic aspects of cryptocurrencies may seem obvious, and cryptocurrency-based games have been anticipated for a long time, in the end they came in different forms of actual play than their prophets predicted. At first sight, the practices of playing cryptogames may align with the ideologies associated with the development of cryptocurrencies and the beliefs of early adopters of 
blockchain technologies. However, algorithms of value creation tend to go askew when they meet the limitations of the real world economy. In this article, we will observe through a case study how play as a cultural practice deviates from the theoretical trajectories set in white papers and the rules inscribed in the cryptogame itself. The object of our study is CryptoKitties (2019), which is an online multiplayer game developed by a team at Axiom Zen (Canada), later reorganized into Dapper Labs in 2017. In this game players collect, breed, buy, and sell different types of virtual cats. It is based on blockchain technology, namely, the Ethereum network (CryptoKitties White PaPurr, 2018). Blockchain technologies ensure scarcity on which the game economy is based. But, as we will notice in the course of this paper, this designed scarcity quickly turns into abundance in the process of collectively playing the game. Even the rarest game tokens become devalued quickly if there are not enough players in the game.

In this article, we investigate CryptoKitties as an example of blockchain-based game design and play. This is important for two reasons. Blockchain technology has been hyped to be the 'gamechanger' in areas like financial services, smart contracts, logistics, secure communication, and governance models (Campbell-Verduyn, 2018; Hütten \& Thiemann, 2018). As digital games are often regarded as an industry where technological advancements first get concretized, it would be logical to assume that blockchains start figuring in a way or another in the development, packaging, and distribution of digital games, and the management of player response to those games, too. The significance of blockchain in this way can be theoretically associated with the rise of digital distribution systems of games, the erosion of boundaries between players and developers, and the growing importance of user feedback in building sustainable gaming infrastructures.

Generally speaking, blockchains are distributed, decentralized, and growing collections of records, or blocks, that are interconnected through cryptographic means and managed by peer-to-peer networks, and that permit secure and anonymous transactions between users (Beck et al., 2017). The most talked-about uses for blockchain technology are cryptocurrencies such as Bitcoin, Ether(eum), Ripple, Litecoin, Monero, and Dogecoin, their variants, and their social and financial implications (e.g. European Union, 2018; CoinMarketCap, 2019). In addition, the technology is speculated to have a significant influence on digital contracts and archives in the near future. Blockchain is an interesting theoretical idea and a source of inspiration for developers, but it also has practical applications many of which are already out there and open for anyone to start using.

As stated, ludic aspects of blockchains are especially visible in the functionalities of cryptocurrency-based cryptogames. In addition to CryptoKitties, there are hundreds of other examples of existing cryptogames (e.g. Cryptopunks, Decentraland, MyCryptoHeroes, HyperDragons, Gods Unchained, Etheremon, Blockchain Cuties, NeoWorld, Axie Infinity). As of 
April 2019, based on open data sources, the number of cryptogames was estimated to be over 650, excluding gambling games. Only a small minority of these games, however, have stable player bases and are able to bring profits to their owners (Tomko, 2019). According to DappRadar (2019), the total number of cryptogames online was 574 on October 20, 2019, and 96 of them had at least one financial transaction in the previous week, with the total volume of transactions varying between 1 and 417,800 in US dollar equivalent.

Oftenmost cryptogames are neither developed by game companies, nor is their design shaped by typical business models or assumptions such as profit-making. Many of them are are merely 'proofs of concept' (Tomko, 2018; 2019), often to get seed investment. Analytics services such as DappRadar (2019) inform us that blockchains are mainly used for two types of games, online casinos and collectibles. This is because the checking transactions in distributed online peer-to-peer networks are relatively slow, so any transaction in the game, from a trade to simply cancelling a trade, can take from minutes to several days depending on the fee and network load (see ETH Gas Station, 2019). As it stands, blockchain technology does not seem applicable for the design of the most popular game genres such as first-person shooters or real-time strategy, although several attempts have been made in this direction (e.g. EOS Knights, HyperDragons, Epic Dragons), and many more are likely to follow, as hybrid blockchain apps and various 'sidechains' are being developed further.

In order to understand how blockchains can be used for game design and play, we analyse specific characteristics of value created through digital scarcity and blockchain-proven ownership in cryptogames, using CryptoKitties as a case study. Compared to other cryptogames, CryptoKitties has the longest and most consistent market history, instantly accessible in its totality as open data comprising all transactions on the blockchain, which allows at least some generalizations. The more detailed objective of this paper is to demonstrate the limits of scarcity in value construction (valuation) for owners of CryptoKitties tokens, manifested as breedable virtual cats. In the end, through this analysis we are going to take a look into the economic ramifications of cryptogames as well as their social implications and importance as showcase pieces for non-fungible tokens, or NFTs.

Our work extends the trends set out by earlier cryptocurrency studies from the perspective of cultural studies. So far, blockchains have been approached in research through a range of domains, most of which have been either technical in nature, or about the most prominent uses, i.e., about Bitcoin (e.g. Yli-Huumo et al., 2016; Beck et al., 2017). Some work has also appeared that deals with the potential economic implications of the technology beyond just cryptocurrencies (e.g. Hütten \& Thiemann, 2018). In addition, there are leisurely uses that the blockchain technology is 
envisioned for (see Chohan, 2017), but research on these is still in its infancy. Similarly, very little academic research on cryptogaming exists as of yet (Scholten et al., 2019, being the key exception), which is why we are also relying on media coverage, crypto trading data, and information from cryptogame developers and players in this article.

Practically speaking, we use open blockchain analytics such as DappRadar and Etherscan, as well as player-created analytics, supported by observations from documented one year-long participation in the said game (Середа, 2019) in our study. Combining theoretical cryptocurrency and Bitcoin studies, open data analysis, and virtual ethnography enables a grounded discussion on the opportunities and business potential of blockchain-based game design. In this article, we first take a look into how the in-game economy of CryptoKitties works by contrasting its functionalities to those of the Etherium blockchain and the cryptocurrency Ether. After that, we study how value is created in CryptoKitties and other cryptogames. Towards the end of the article, we discuss certain shortcomings of blockchain-based game design and play. This opens up new perspectives for research and improvement of this field of study in the future.

\section{CryptoKitties: the Money Game}

Since November 2017, CryptoKitties has proven to be one of the first still existing and, so far, one of the most successful cryptogames. In December 2017, it was even called 'insanely popular' in the media (Schroeder, 2017) as it attracted about 7,000 players simultaneously at its peak (DappRadar, 2019). In CryptoKitties, players can breed, trade, and gift cartoon cats, and, as of 2019, also use them in raffle-like games of luck with the help of external resources. Another earning mechanic is putting kitties up for sire, so that another player can pay to breed with a specific kitty. Due to its mechanics being based on the Ethereum blockchain, each cat is unique (non-fungible) and cannot be handled by anyone other than its owner, not even by the game's developers (CryptoKitties White Pa-Purr, 2018; CryptoKitties, 2018d). If the cat resulting from breeding has certain high-level 'cattributes', has “fancy" or prestigious appearance, it can be traded with profit (via an auction or for a fixed price) on the game's own marketplace or outside of it, and, recently, even in other cryptogames. The most interesting example of this interoperability (Ferguson, 2019) is a recent collaboration between CryptoKitties and Gods Unchained, in which the players of both games are provided with unique items to add to their NFT collections and to be shared as assets in both game universes (Putney, 2019).

The monetary value of cryptokitties is based on each of them being unique, and on the fact that the blockchain guarantees their ownership. One of the main purposes of blockchains is to prevent double spending and to attribute a clear, non-disputable digital ownership, for example, through 
mining where a user can claim ownership of new tokens contributing with a node. Thus they are particularly suitable for digital collection games where the purpose is to "catch "em all," or to acquire the rarest possible items. Blockchains ensure the validity of transactions and transaction records by resisting the modification of data by design and peer quality control (Campbell-Verduyn, 2018). They therefore enable the kind of artificial scarcity of digital goods that forms the basis of many online economies (Lehdonvirta \& Castronova, 2014). As virtual items are held in value by people willing to pay money and/or time for them, they are market-wise just as valuable as realworld items and services can be, and can be treated as "real" by their owners (Lastowka \& Hunter, 2004).

There are so far seven known cases where the trading prices of cryptokitties have risen to over $\$ 100,000$ (Mala, 2018; CryptoKitties Sales, 2019), some of them raising suspicions of money laundering (Varshney, 2018). Typically, the price of one kitty ranges between a few cents and over a hundred USD, or 0.001 and $1 \mathrm{ETH}$, in special cases reaching as high as $600 \mathrm{ETH}$. It bears noting, however, that the exchange rate of Ether to fiat currencies like USD or EUR can fluctuate heavily (see Fig. 1). On a player-made website, the estimated average sale price of a cryptokitty in US dollars is $\$ 48.50$, and the median sale price is $\$ 7.09$ throughout the game's lifespan (CryptoKitties Sales, 2019). However, these numbers do not reflect the gigantic changes in ETH exchange rate between 2017-2019, which only vaguely influence the game itself. Indeed, as we will see later, prices that are originally in ETH, converted into real-world currency, indicate very little about the in-game value of a cryptokitty as a game token.

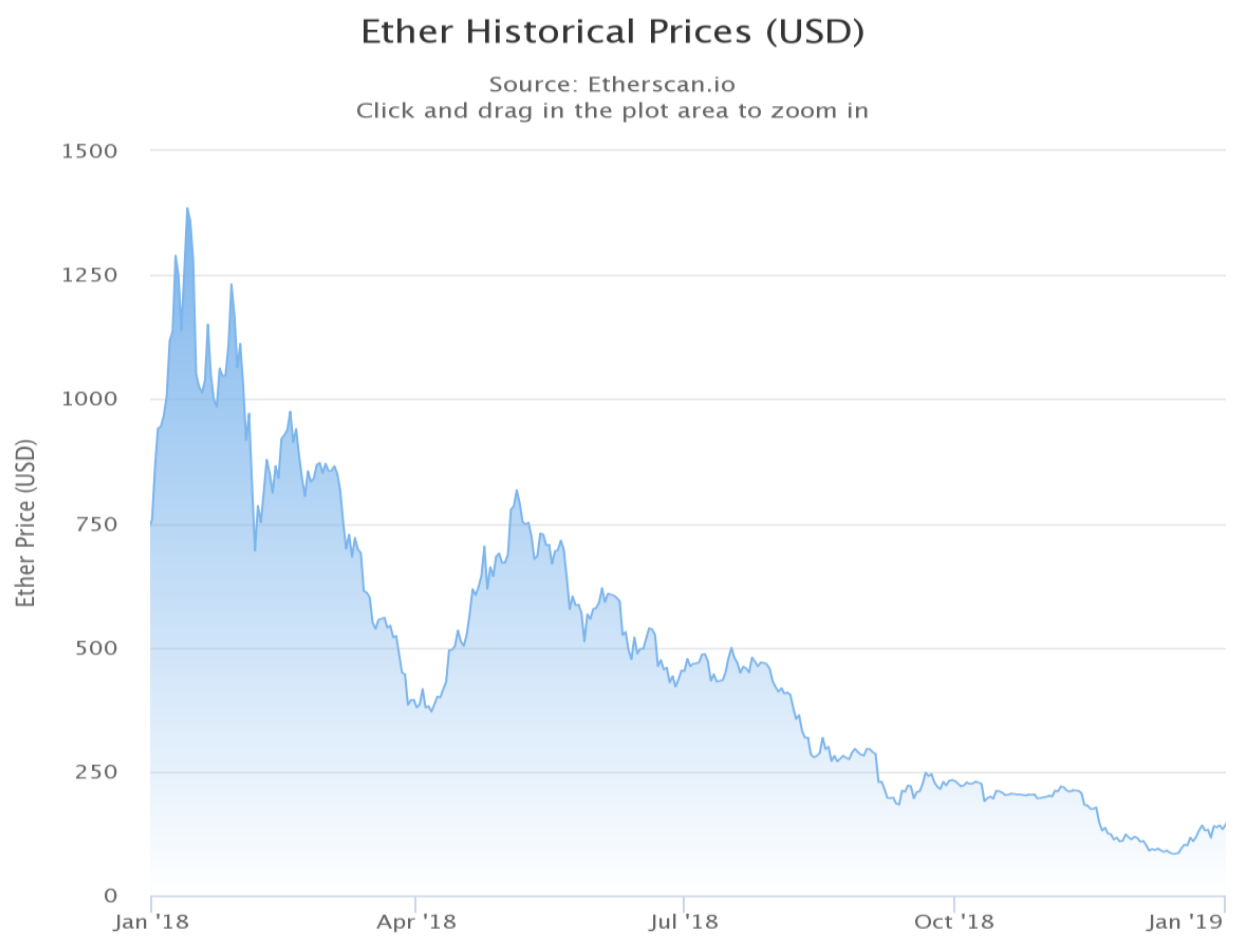


Figure 1. The development of the price of Ether between Jan 2018 and Jan 2019 (Etherscan.io 2019).

Originally, cryptokitties are valued and traded in Ether (ETH), and their principal market characteristics are shaped by the Ethereum platform and the hypervolatile currency that is tied into it. For this reason, they can be investigated from the viewpoint of 'platform studies', which helps us decipher the ways the platform is shaping the economic and ideological aspects of the game (see Gillespie 2010). Originally, the ideology of a cryptogame builds on the libertarian claims of cryptocurrency adopters, as 'techno-libertarian beliefs' (Hütten \& Thiemann, 2018) are embedded into the coding of Bitcoin, the first and most well-known cryptocurrency to date. These convictions stem from the foundational values of the internet itself - freedom, liberty, autonomy - as well as the 'cyber-libertarian' thinking on a larger scale that has characterized the development of internet services and platforms as well as the "virtual economy" discourse especially in the pre-Web2.0 era (e.g. Castronova, 2007; Penney, 2012). While all players start in 'abject poverty' in the speculative virtual economy of the 2000s (Castronova, 2007), in the blockchain-powered cryptocurrency world, professional cryptotraders start big, and casual players have to rise up from 'cryptopoverty' first, if they are able to rise from it at all. Virtual worlds, and virtual markets especially, are currently not the fair and equal meeting grounds they were once thought to be.

Cryptogames rely on the functions of the cryptocurrency platform to the extent that it makes no sense to omit the investigation of the platform in mapping out how the game itself is designed to be played. Also the game mechanics of cryptogames entirely depend on the functionalities of the cryptoplatform upon which they are built. For instance, the in-game value of objects (here, cryptokitties) cannot be dissociated from the real-world value of those objects, as it directly affects the ability of players to engage with the game. Cryptocurrency is therefore an extension of the technological blockchain platform that enables the exchange of tokens and constitutes the economic level of a cryptogame. It has to be noted, however, that for the needs of this article, we stay within the perspective of a single platform, Ethereum, and thus our investigation cannot be generalized to encompass all existing or future cryptoplatforms.

In practical terms, we focus on Ether as the in-game currency for CryptoKitties - the function which it served best throughout 2018 when compared to its real world performance. During our research, the real world value of ETH decreased tenfold in the first year of the game's existence (see Fig. 1 for details). But even then, based on our observations and play experience, at virtually any moment in the game, one ETH $(\$ 130-1,300)$ would still buy enough valuable cryptokitties to carry on with the game and make (potentially real-world) money in it by breeding kitties and engaging in speculation about their prices and future investment value. This has also been noted in CryptoKitties 
Investing Essentials (Pranked, 2018), written by one of the most active players, even though this specific piece of advice is not valid anymore due to market changes in 2019.

The big crash of Ether in 2018, commonly referred to as the 'bear market' by cryptotraders, had a surprisingly modest and largely positive effect on the in-game economy. During CryptoKitties' first year, the volume of sales in Ethereum was fluctuating within a reasonable range in the game, and roughly doubled in the second half of the year due to various in-game events, such as the introduction of 'Family Jewels', which changed the value of many kitties on the market (Henrie 2018), and the introduction of new designs of 'purrstige traits' (Cryptokitties, 2018a) (see Fig. 2 for details). New genes are not introduced anymore, as they are supposed to be scarce, and there is a limit to them hardcoded into the game system. Still, new visual traits are continuously introduced to keep the game going and to help maintain the market active.

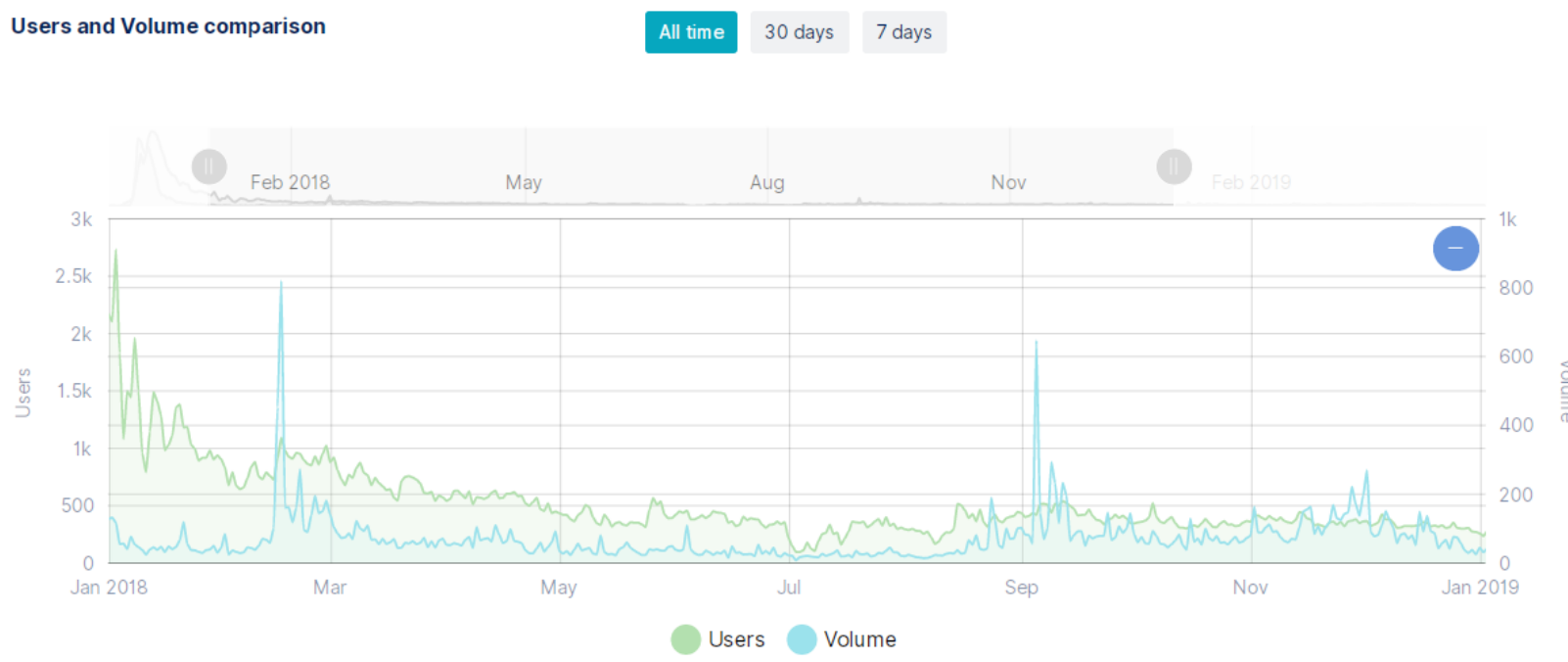

Figure 2. The relative amount of players of CryptoKitties and the volume of kitty sales between Jan 2018 and Jan 2019 (DappRadar, 2019).

\section{Game as a Market: An Outline of Cryptokitties' Economy}

One of the most creative features of the CryptoKitties game system is that it was not introduced at once, in its finished and comprehensive form. Instead, the game gradually transpired by the combined efforts of developers and players starting from going public in November 2017, until November 2018, when the so-called 'Kitty Clock' stopped and the last cat from the pre-defined gene pool of 50,000 so-called 'Generation 0' kitties was issued by the developers (CryptoKitties, 2018a). This "participatory design" approach is also visible in the economic aspects of the game, as we will later demonstrate (see Liu, 2017). Genes and traits characterizing the kitties were introduced one by one within this first year of the game's existence until they created a complicated binary system that included four levels of 10 basic traits ('cattributes', such as base, highlight, and 
accent colours, pattern, mouth shape, eye colour and shape) which could be bred from Gen 0 traits. This system can be seen as an add-on to the game interface now, and is included in the official guide (see Fig. 3 for an excerpt), but originally its intricacies were left to be discovered by players who would gradually study the open source game code available on the blockchain to decipher its logic. Based on this information, the ultimate goal of the game is to breed a 'queen bee', as players sometimes informally call it, - a kitty whose 'cattributes' are all level 4.

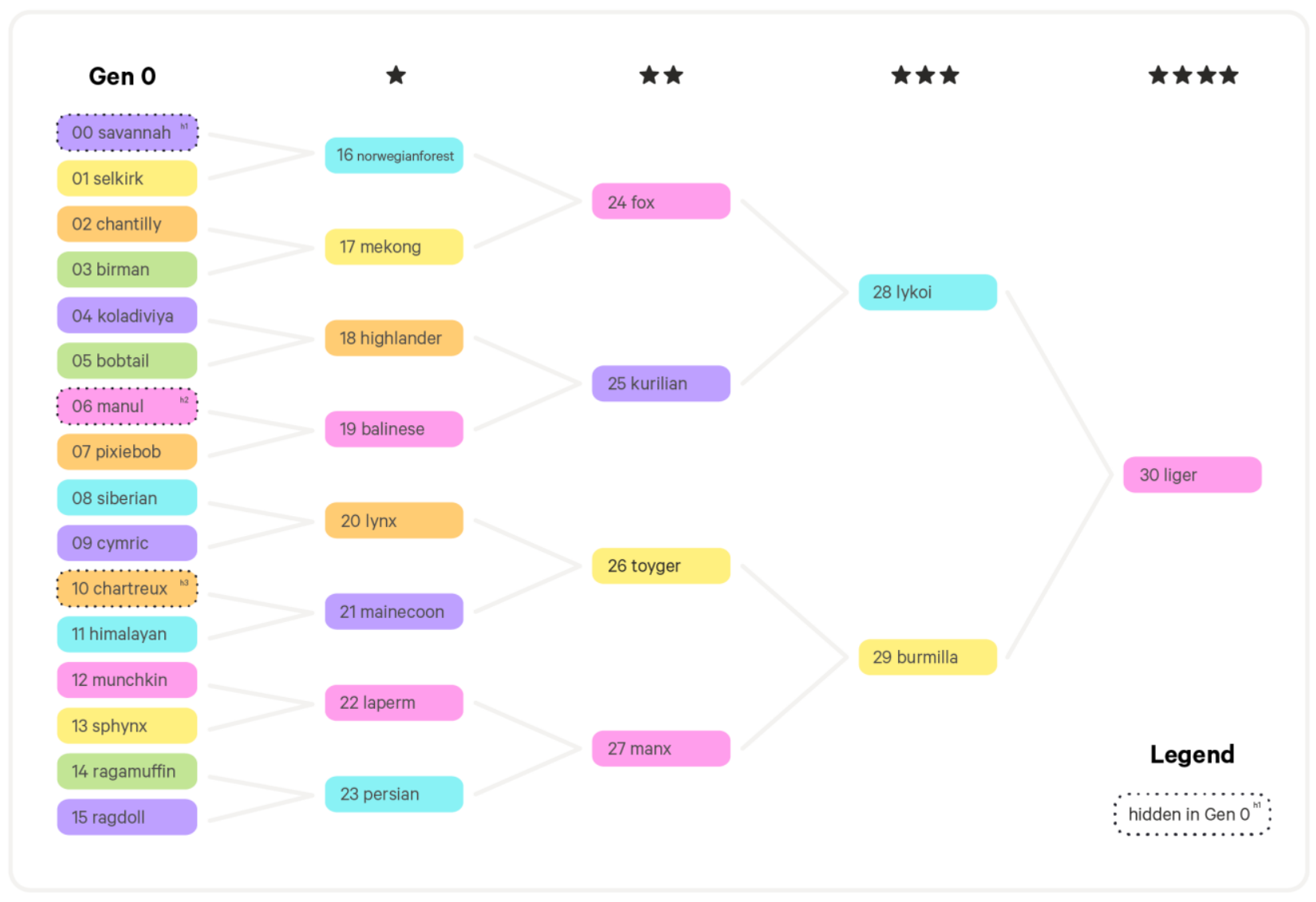

Figure 3. A genetic guide for all varieties of cat fur from the official guide (CryptoKitties Guide, 2019).

The biggest fall of the cryptomarket coincided with the most active time in the game before November 30, 2018, which was the day the developers stopped minting new Generation 0 kitties. Since then, new kitties only appear in the game as a result of breeding (CryptoKitties White PaPurr, 2018). According to player made statistics (Kitty Explorer, 2018), the median price for a Gen 0 kitty was decreasing after two peaks, December 2017 and February 2018. In the spring and summer 2018, it stagnated to below 0.5 ETH and fell below 0.1 ETH in April, then started rising again in October, fluctuated around 0.7 ETH towards the final day of their creation (Nov 30), and then slowly went down to $0.25 \mathrm{ETH}$ (see Fig. 4 for details; here, the median price is more 
illustrative a factor of the fluctuations of cryptokitty prices than the average price, because of the broad range of values).

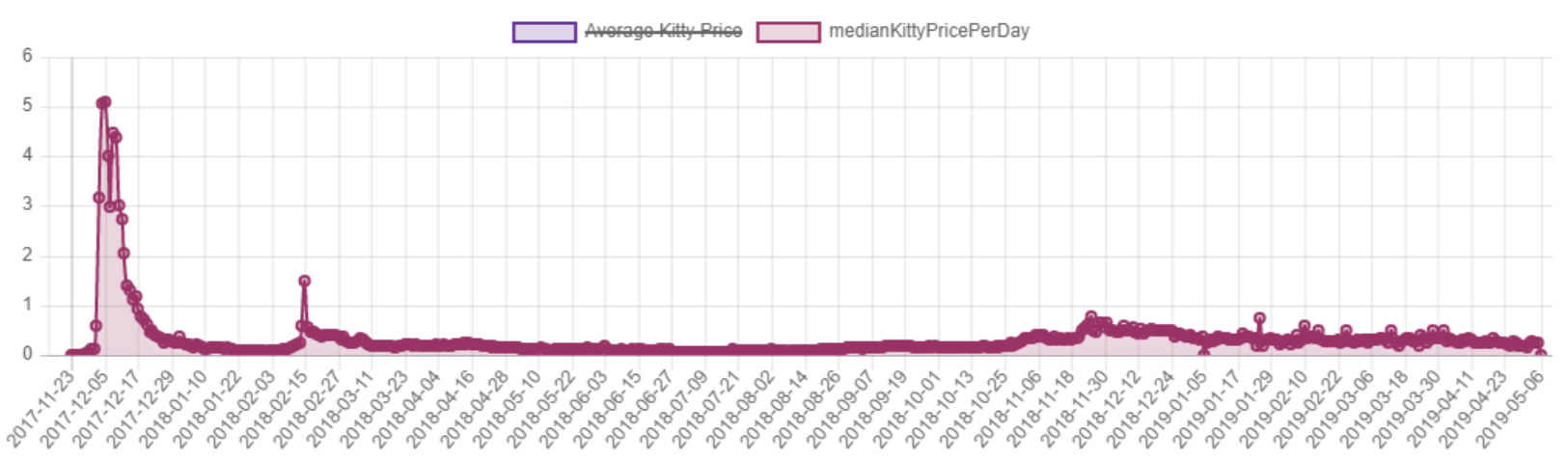

Figure 4. Median cryptokitty price in ETH per day between Nov 23, 2018 and May 6, 2019 (Kitty Explorer 2019).

As mentioned, the game economy of CryptoKitties is based on the limited amount of Gen 0 kitties, whose value was thought to inevitably increase after the developers stopped minting them in November 30, 2018 (CryptoKitties, 2018c). As we can see from the figure, this did not happen: the price of kitties has been gradually decreasing since the November peak. With little more than 89,000 players signed up in the entire history of the game, and only a few thousand active players (according to KittyHelper, the total number of active players in the game was around 5,000 in the third quadrant of 2019), 50,000 Gen 0 kitties seems too large an amount to consider them 'scarce'. In the first half of 2019, it was still possible to find a cute and relatively rare Gen 0 kitty for sale for about $1 \mathrm{ETH}$, or to make a direct trade with its willing owner. The prices have slowly been drifting downwards, although the range of prices has been too broad and the number of sales too low to make definitive statistical observations. In October 2019, the cheapest Gen 0 was on sale for 0.33 ETH, which is 0.0079 ETH cheaper than its initial cost.

Originally, CryptoKitties developers announced that only 50,000 Gen 0 kitties would ever come into existence, but they have not even released this quantity. There were 38,017 Gen 0 kitties in the game as of October 15, 2019, and 2,918 of them (about 8\%) were on sale. The remaining 10,000 or more Gen 0 kitties are expected to be released when there are more players in the game. During the following year after the Kitty Clock stopped, the number of daily and weekly players has only been decreasing (KittyHelper, 2019). This can be seen as another failed adoption cycle: the game did not succeed in generating enough traction for new adopters, and, after a relatively short organic "heatup", the in-game market gradually went "cold" again. Why does the in-game economy seem not to work? One possible explanation to this is, relying on the platform infrastructure: As the mass adoption of cryptocurrencies has so far been based on wide-spread speculation about sharply 
ascending prices - and high volatility at times, making investing seem lucrative - the same mechanics have not been activated in CryptoKitties due to the relative stability of prices on the ingame market.

In addition to the discrepancy between the price of kitties in ETH and their valuation in the game, another interesting factor is that the in-game value of kitties in ETH is not connected to the real world price of Ether, either. This could be explored further as a circular dependency. Both the company's marketing efforts and the 'bear market' condition were just enough to keep the game stable; the only huge peak of adoption happened right after the official launch. As we can see from the next figure (Fig. 5), the average daily number of CryptoKitties players has been fluctuating within the range of 200-500 for the most of the game's existence (see DappRadar, 2019), which is minuscule compared to many other multiplayer online games. It could be speculated that the number of players might have been positively affected by the Ethereum market crash, as the game became "more affordable" - provided that there was existing interest in this kind of cryptogaming in the first place. However, it seems that such vital metrics for online multiplayer games as the amounts of daily and weekly players were not growing significantly within the first year, which means that new player adoption would only compensate churn.

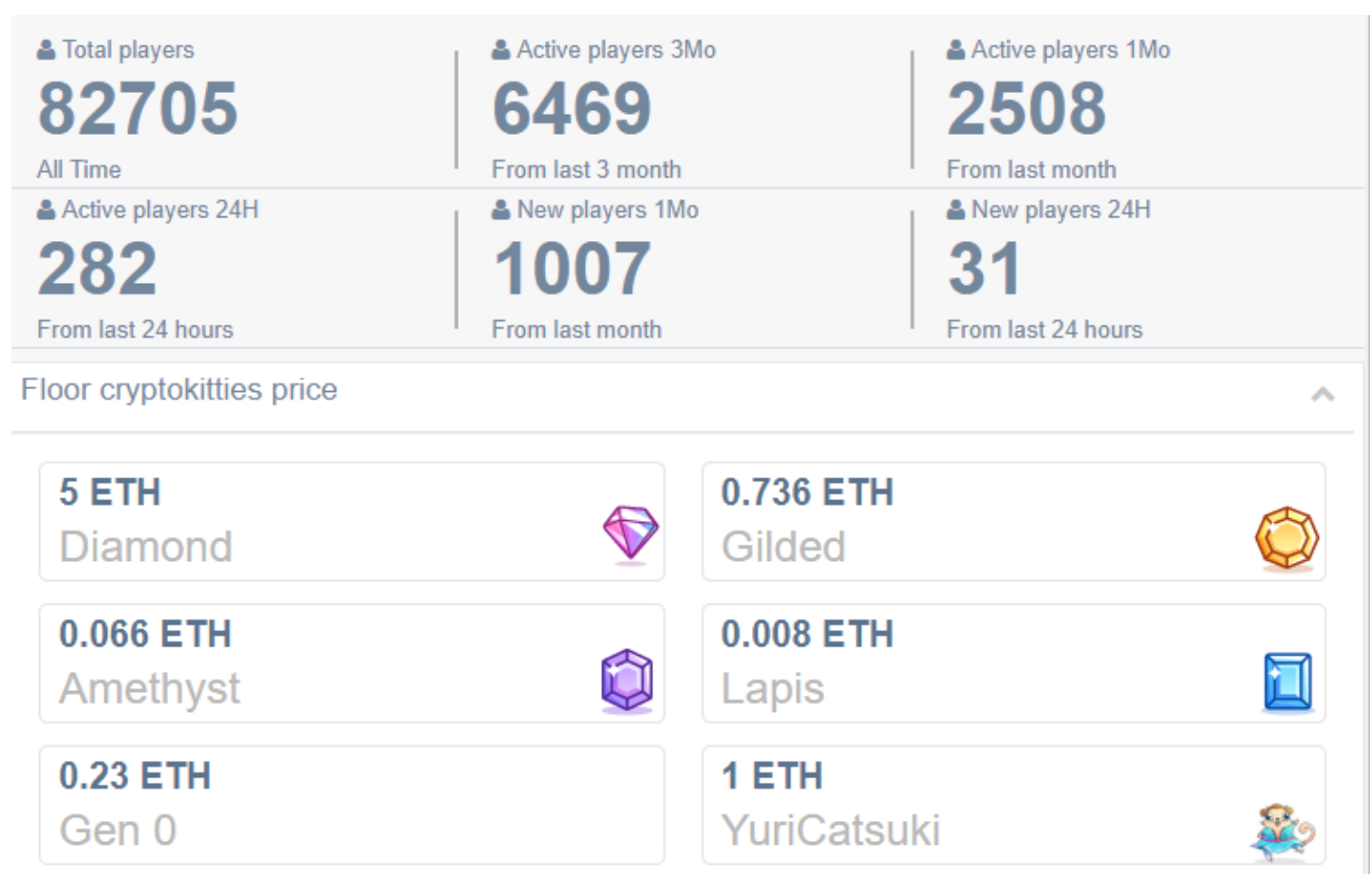

Figure 5. An excerpt from CryptoKitties statistics on May 5, 2019 (KittyHelper, 2019).

At the time of writing (Autumn 2019), the number of kitties is growing and the in-game value of single cryptokitties, reflected in their prices on the market, is gradually decreasing. The game 
economy and the game mechanics of CryptoKitties were originally based on 'digital scarcity', but this has not proved to be a viable strategy for making the game work. For instance, the game mechanic of breeding new kitties automatically reduces the scarcity of certain traits and hence their value (Liu, 2017). Breeding and trading kitties, however, are vital activities from the perspective of gameplay, and the player's interest in taking part in them lies in the possibility of increasing the (speculative) in-game and out-of-game value of the kitties owned. In order for the game to work then, in economic terms, there should be clear incentive for the player to both take part in the multiplayer game (=to have "fun", etc), and to be able to prosper through their ownership (=to see the value of their in-game possession increase out-of-game).

From the perspective of gameplay, it is also slightly problematic that all the basic activities cost money, although they only cover the costs of mining for a new kitty and do not generate any profits for the developer company. This is a clear downside of a digital game based on blockchain technology. In effect, the players of CryptoKitties are paying for the maintenance of the game (CryptoKitties, 2018b). Transaction costs are likely to discourage new players from taking part in the game, and from continuing play in the long run. There are also other inconsistencies in the game. For instance, the precious stone symbols or 'Family Jewels' that can be seen in Figure 5 above were originally introduced in February 2018 as an inheritable reward for kitties that discovered new, "enhanced" cattributes, or 'mewtations'. After a year, they are supposed to somehow influence the price of a kitty in addition to factors such as its generation, rarity of specific cattributes, and visual appeal. However, there is always a considerable supply of them on the market as some high ranking players specialize in breeding kitties with gems for sale, while nothing can be said about demand.

Like other cryptocurrency researchers who study cryptomarkets from within, we deliberately do not focus on questions concerning the "real-life", market value of Ether, its 'wildly fluctuating values' (Campbell-Verduyn, 2018), speculative manipulations (Garcia et al., 2014), and yet-to-be-explained hypervolatility. Subjective assessment of future returns (Garcia et al., 2014), partly fed by the urge to gamble, is an irrational element in both CryptoKitties and the so-called 'Bitcoin game'. Within the confines of this article, we investigate the mechanisms through which value is created in CryptoKitties from three viewpoints in the following section: First, by analysing how blockchain functions as the basis for valuation; second, what role rematerialization plays in it; and finally, how the (pseudo) true ownership of tokens is tied to value in cryptogames. 


\section{Value Creation in CryptoKitties}

The path towards understanding the value of assets in cryptogames starts with a more general question of how the value of cryptocurrencies is constructed. Furthermore, in order to grasp how cryptocurrencies work, we need to ask how the value of any currency is constructed, especially in today's digitalized and globalized world. As a background for our treatise of cryptogaming, we are going to cast a brief look into the sociology and communicative aspects of money next. Once we see how cryptocurrencies work in terms of valuating things, we can move on to examining how value is created within and around CryptoKitties and other cryptogames.

Blockchain as the Basis for Valuation

Money in general, and electronic money in particular, has been interpreted as a semiotic system that is gradually becoming more and more detached from its early material signifiers (such as the gold standard) or commodities (hence, the term 'commodity-based money' as opposed to purely symbolic tokens) (e.g. Parsons, 1963; Ganßmann, 1988). Transforming real-world monies into digital currencies and then into cryptocurrencies has seemingly continued this trend, complying with Baudrillard's (1996) concept of 'electronically mediated hyperreality'. Nevertheless, the temptation to see cryptocurrencies as the ideal case of 'networked', 'disembedded' or 'hyperliquid' money is compromised by acknowledging the real-life conditions and technical limitations of their usage. In this part, we discuss two of these limitations: the transaction costs or the 'gas price' of using the blockchain, and the expertise required to perform operations on it.

The so-called 'gas price' (Gwei) is the first major contradiction to the popular claim about fast and feeless transactions and 'nearly instantaneous exchange' (Campbell-Verduyn, 2018) of cryptocurrencies. Simply put, this is the fee paid to miners for computing new 'blocks', or smart contracts in the blockchain. The possibility of instantaneous transactions is one of the basic requirements needed in order for cryptocurrencies to be defined as money. Unfortunately, cryptocurrencies do not function smoothly for instantaneous transactions at the moment (Baldwin, 2018; Lamison-White, 2019). Literally every activity in CryptoKitties costs money, including giving a kitty away for free or cancelling its sale. Each action on the blockchain requires a complex computational process that needs to be paid for in 'gas'. The fee is established on the basis of an auction, where each player sets their 'gas limit', or the maximum amount of units of gas they are willing to spend on a transaction. The transaction fee itself consists of this gas limit times the 'gas price' that the player sets to pay per unit of gas. Gas is thus another word for transaction costs that in the Etherium blockchain are paid for in Ether that is transferred directly to miners who enable the transaction in the first place by their computation power (MEW Help Center, 2018; LamisonWhite, 2019). 
In practice, the price the player is willing to pay affects the mining time of the transactions required (MEW Help Center, 2018; Lamison-White, 2019). Thus wealthier players can pay more to speed up transactions, while players less eager to spend their Ether on gas sometimes have to wait for hours for a basic transaction in the game to resolve. Also, the fee increases with the total number of simultaneous transactions in the game, which means that the game is simply dead stuck for less wealthy players during big in-game events. In the worst case, the whole Ethereum network becomes clogged (Lamison-White, 2019). This happened in the first days after CryptoKitties was launched, and all transactions were slowed down or even halted (Cheng, 2017) regardless of how much 'gas' was being offered to miners. With the obligatory gas fees going to the third party (cryptocurrency miners), the game would always be less than a zero sum game, even if everyone was playing fair which is not the case.

The second contradiction of cryptocurrencies is the vast amount of technical and operational skills needed to understand and use what was designed as a universal payment method. According to a survey (Ponomarev, 2019), backed by industry practitioners (Tomko, 2018; 2019), the biggest obstacle in cryptocurrency adoption is 'onboarding', or overcoming the technical difficulties related to owning and operating a crypto wallet. We assume that the overcomplicated user experience was seen as a necessary evil by crypto traders who have been internally motivated to use crypto wallets and buy cryptocurrencies, being attracted by the probability of high financial gains. Gamers, on the other hand, and especially casual gamers, whom cryptogames are targeting now, are used to much friendlier game interfaces and welcoming ludic affordances. Many of these players were disappointed and even pushed away when they first encountered the many quirks of crypto wallets, including the constant threat of a lag, unpredictable gas fees, the impossibility of canceling transactions, and especially the fact that a lost account in a crypto game could not, by any technological means, be recovered.

Both of these contradictions are rooted in the most essential elements of cryptocurrencies, mining and blockchain. Without these, cryptocurrency is just another electronic money, and a game based on it is just another digital game with its own economy and regular in-game currency (see also European Union, 2018). Therefore, in order to understand how cryptogaming is developing, we need to recognize the characteristics and challenges of cryptocurrency that follow from the procedure of issuing it (mining) and the way it is organized and kept track of (blockchain). As we have seen, in addition to their assumed 'hyperliquidity', cryptocurrencies have material and physical characteristics that define the games designed to be played on the blockchain. 


\section{Materiality of Transactions}

Defining the value of cryptocurrencies through mining brings back the physical, real-world aspect of these currencies, making them something more than just electronic money. Paradoxically, "Bitcoin rematerializes money" (Garcia et al., 2014). Mining as a metaphor for issuing cryptocurrency was first used in the Bitcoin White Paper (Nakamoto, 2008). Interestingly, this original document also mentions gold as the value standard, as well as its limited supply as the basis for value construction: "The steady addition of a constant of amount of new coins is analogous to gold miners expending resources to add gold to circulation" (Nakamoto, 2008). As Maurer et al. (2013) have argued, this mining metaphor is a deliberate reference to earlier, precious-metal based monetary systems, and as such it might be another extra-technological factor that has stimulated Bitcoin adoption and its acceptance by the general public.

Here we can see how a connotative meaning associated with a technical term implies certain social interpretation of a technological system. Cryptocurrencies are conceived to be more 'material' as their users make an emotional connection between the invisible digital work of an algorithm and the painstaking process of gold mining (Calvão, 2019). This new materiality of cryptocurrencies has been labeled, for instance, 'digital metallism' (Maurer et al., 2013). According to this interpretation, it is both a semiotic and a financial feature, which is crucially important for our goals, as we are looking for main principles of value construction where these two areas overlap. The materiality of the mining rigs and the electricity consumed by them justifies cryptocurrencies as a "currency deriving its value from the material out of which it is made" (Maurer et al., 2013), and hence, commodity-based money.

The metaphor of mining has also inspired the narratives of many cryptogames (e.g. Ether Kingdoms and MyCryptoHeroes). In CryptoKitties, both human and machine labour needed to compute the transactions on the Ethereum network make the materiality of the tokens, cryptokitties, discernible. The materiality of a cryptokitty is also emphasized by its developers and owners through a variety of visual metaphors that mark it as a special token that exists in time and space, almost as if it was a "living thing" rather than a string of code. Every kitty is unique and can be named according to the wishes of its owner. A kitty is born from an egg, and the player has to click it several times to activate birth. This process is the most symbolic ludic moment in the game, as there is always an element of surprise in the birth of a kitty - an element of surprise that makes it interesting to stream the predominantly static game on Twitch, not unlike the genre of 'unboxing' videos on YouTube. Developers claim that the birth of a kitty is the most demanding part of the game in terms of the computing power on the blockchain, and this is also the reason why the birthing fee is so high (CryptoKitties, 2018b). 
This takes us back to the initial question of meaning. Just as the value of cryptocurrency is both a technological (material) and semiotic construction, the same can be said about a token in a cryptogame such as CryptoKitties. Unlike cryptocurrency, it is also part of a higher level semiotic system of game rules, defined by its platform (Ethereum), its own cryptocurrency included, but also freely interpreted by its players. For instance, it is common knowledge among players that the value of cryptokitties depends on their appearances (cakepie99999, 2017; Pranked, 2018). As participatory netnographic research suggests (Середа, 2019), the value of a cryptokitty should be estimated through taking all these different elements into consideration: technology, including its 'material' aspects of mining (the 'rigs', fees, and lags); the platform, including the specifics of the blockchain-powered cryptocurrency; the complicated semiotic system of game rules; and, finally, player behavior on the market, in game communities, and in the game. Like in any other game, this behavior can be either fair and social, or unfair and disruptive, or anything in between. In addition, like in most multiplayer games, cheating is always a viable option.

\section{(Pseudo) True Ownership of Tokens}

Player-owned content is often seen as one of the core values of cryptogames (Tomko, 2018; CryptoKitties, 2018d; Ferguson, 2019). "No one can take it away from you" is a recurring slogan in the crypto world (e.g. CryptoKitties White Pa-Purr, 2018). Nevertheless, within the cryptogame discourse, the one who might want to rob players of their digital assets is a person or a company who is invested in the development of these assets. The claim of true ownership is thus made against 'unfair' game publishers and "developers influencing the ecosystem and larger economy" as a source of insecurity for players in CryptoKitties White Pa-Purr (2018), indirectly implying Blizzard Entertainment. The global state of the game industry provides many reasons to be critical of them - the Blizzard End User License Agreement (2018) is usually quoted as an example of denying players' ownership of game items.

Blockchain-based tokens and assets are stored in players' wallets and they are tradeable as an open market exist for them. In CryptoKitties, this market is founded on the idea of non-fungible tokens that can be individually owned, traded, and bred. Generally speaking, transparency of data on a blockchain is the basis of trust in so-called "trustless" anonymous transactions of cryptocurrencies a principle that is already visible in Nakamoto's White Paper. Both anonymity and trust, however, are limited by 'exit points' into the real world economy, as selling cryptocurrency for fiat money de-anonymizes their holder except when fiat money is transferred as cash (that is supposed to be made obsolete by the very idea of electronic money). The materiality of fiat money adds another problematic level of trust and safety to such transactions: withdrawing cryptocurrency into cash is not as straightforward as one might think. It involves using specialized cryptocurrency exchange 
services that are imposed to the same legislation as banks (Dob, 2019), the transactions are most vulnerable to robbery, and likely to be considered a 'grey' or 'black market' operation in most countries.

Trustless anonymity in crypto token exchange and its eventual compromising can be likened to the state of ownership in networks powered by blockchains. Based on research conducted as early as 2011-13, Maurer et al. (2013) suggest that the protocols of Bitcoin "offer not anonymity, but 'pseudo-anonymity"'. Similarly, Campbell-Verduyn (2018) refers to cryptocurrency users as “quasi-anonymous individuals". Just like Bitcoin paradoxically was long perceived as a guarantee for solidity, materiality, and stability (Maurer et al., 2013), the idea of 'true ownership' of in-game objects is more an answer to certain social anxieties than a fulfilment of user needs.

Surprisingly, the model of ownership offered by crypto games demonstrates similarities to ownership of a (semi-legal) physical object. Even if blockchain minimizes "exploitation" from the side of game publishers, it does the bare minimum to protect ownership rights from perpetrators, hackers, and cheaters. Apart from advanced technological security measures, which still easily fail the human factor, there is very little legal protection against 'kitty burglars' (as a notification message in CryptoKitties calls them). Finally, a lost crypto wallet cannot be recovered, and in this case, all in-game tokens are also irreversibly lost. In an obvious attempt to deal with this anxiety, in April 2019, the company initiated a migration of cryptokitties to their proprietary cryptocurrency wallet Dapper (CryptoKitties, 2019), somehow protected from loss with the phone number verification and thus deanonymized. Even though a crypto wallet cannot be physically destroyed, 'true ownership' is always endangered by its permanent loss (and eventually set back to centralized institutional support).

In an attempt to clarify the terminological mess with regards to the issue of ownership in blockchain-powered systems such as CryptoKitties, we offer the concept of pseudo true ownership. Through it, we want to illustrate the technological and juridical challenges related to acknowledging ownership in a system that is supposed to guarantee complete anonymity. Legally speaking, the execution of 'true ownership' rights is very limited (hence the 'pseudo'). Blockchain can be used to trace the owner, but, under normal conditions, it does not connect digital property to a specific person in the material world, as this would undermine the anonymity principle. This means that the owners of crypto assets can only claim their ownership by sacrificing their anonymity, and only under the jurisdiction of a few countries where blockchain is recognized as a means to claim ownership in the first place (see Campbell-Verduyn \& Goguen, 2018, for a review of blockchainbased financial activities under different jurisdictions). In some cases, even in a country like China, where trading cryptocurrencies is prohibited by law, cryptocurrency owners can report theft of digital property and hope for legal protection (Madore, 2018), but no such cases have been 
registered for cryptogame assets yet. Not many precedents in court have been created even with conventional in-game assets so far (a brief outline of legal suits related to in-game items can be found in Packard, 2010). To sum it up, in the cryptogaming world, a player does not need an 'identity' to own a traditional crypto wallet - in fact, many players of CryptoKitties own more than one wallet - but it seems that the omittance of online identity markers also undermines ownership.

\section{Discussion: Scarcity and Valuelessness in CryptoKitties}

It is often stated that the value system of cryptocurrencies is based on a fixed supply and predictable scarcity of fungible tokens (see Garcia et al., 2014). Technologically and economically speaking, Ether is no different from Bitcoin in this regard, and Ethereum-based cryptogames highlight scarcity in their white papers and rely on it in their game design systems. The developers of CryptoKitties aimed at innovating with digital scarcity within the confines of the blockchain space (CryptoKitties White Pa-purr, 2018). Initially auction-based, the game sought to project demandbased value through effective exchange prices of its tokens (still leaving their 'material' value, i.e. the mining cost, out of this ideal picture). The supply of these tokens was fixed and their scarcity was anticipated, and their attributes were strategically planned for a game timespan of one year. In effect, the actual price for most tokens in the game fell below their 'material' value after one year, resulting in 'negative value' of most newly bred kitties, as breeding only caused losses to the majority of players.

Scarcity is an important theoretical safeguard of value, but it only works in practice when demand significantly overpasses supply, which is not yet the case in any cryptogame. In May 2019, roughly 82,000 all time players (or wallets) own 1.7 million unique tokens in CryptoKitties. At least 90 per cent of these players are not active anymore, and thousands of tokens are being sold below their net price. Still, the core audience of 'crypto whales' persists and seems to enjoy the game, consistently creating a significant daily trading volume of over 30 ETH in the first half of 2019, which decreased nearly twice in the third quarter of 2019 (DappRadar, 2019), which is a rough equivalent of $\$ 5,000$. As there is an absence of new players, in CryptoKitties in particular and on the market of cryptogames in general, digital scarcity has quickly turned into digital abundance. 


\section{Great-value Kitties}

Purrfect for beginners! These kitties are low-cost, low-gen, have speedy cooldown time. Great for breeding and expanding your Kitty collection!
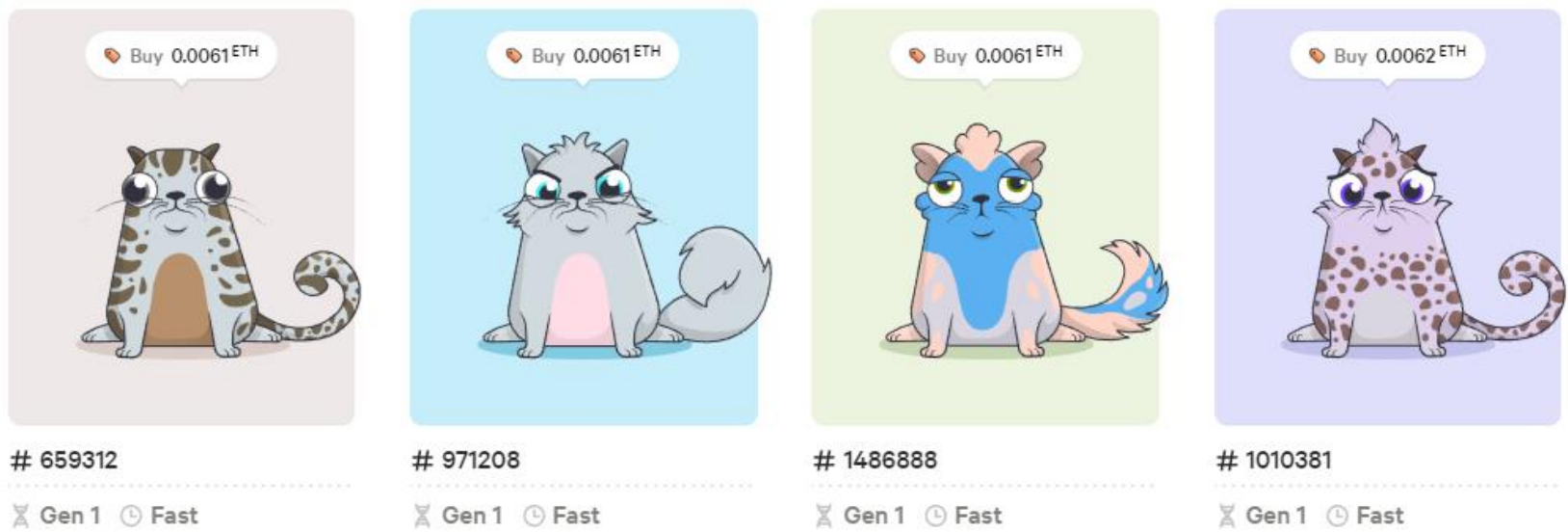

Figure 6. The starting section on the game's web interface on May 5, 2019 (CryptoKitties, 2019).

From the viewpoint of an experienced player, the 'Great-value Kitties' advertised on the front page of the game (see Fig. 6) are basically valueless. They sell for less than the breeding fee or their 'material' mining cost that has been fixed at 0.008 ETH since December 2017 (CryptoKitties, 2018b). Also, the criteria of including them have changed from Gen 3-5 in January 2019 to Gen 1 in May 2019, which means that Gen 3-5 kitties are valueless now, if they do not possess rare traits. For reference, in 2018 speculation with Gen 1 kitties was still a recommended way to make money in the game (Pranked, 2018; Bogatyy, 2018). Now many of them have negative price (less than their net price). According to Kitty Explorer (2019), the median price for Gen 1 kitties was also gradually decreasing after February 2018, as it fluctuated between 0.01 and 0.015 ETH from May to November and fell below the birth price after November 30, 2018.

Breeding a valuable kitty usually takes several attempts, as it is a gambling game, and the shrinking core audience is both enjoying this mechanic and can afford it. As more kitties with similarly valued traits are constantly created by "fancy-chasing" crypto whales, the price of regular kitties will necessarily go down in Ether. This introduces a new concept in cryptogaming which apparently no one has thought about before: valuelessness. At the moment, it looks like cryptogame markets are becoming oversaturated with valueless objects, CryptoKitties being the obvious example here, and most developers do nothing to ensure the obsolescence of these tokens. It seems as if developers are hoping to create new ways to construct value of old, indestructible, and quickly devaluing tokens through game design. Successful results of these efforts are yet to be seen. 


\section{Conclusion: Why Does the Cryptogame Economy Not Work?}

In this article, we have analysed value created through digital scarcity and blockchain-proven ownership in cryptogames, with the Ethereum-powered CryptoKitties game as our case study. Even if cryptogaming may still be an emerging - and currently over-hyped - trend, some games, CryptoKitties among them, have in the past year garnered significant financial interest. When the game design project was reconfigured as its own company Dapper Labs in 2018, it was able to raise $\$ 12$ million in venture capital, and later that year another \$15 million (Paez, 2018). At the time of writing this article, at least 1.3 million virtual cats have been bred by CryptoKitties players. Even though the game might not be an economic success story (as of yet) (see Wood \& Lindman, 2018), it has been so popular that it has at times significantly slowed down the entire Ethereum network (Cheng, 2017). CryptoKitties is one of the first applications of cryptocurrencies with which it has been possible to gamble for profit, but which actually did not appear as stock trading but as a fun game instead (Nathaniell, 2018).

This article has been aimed at establishing an understanding of why blockchains are important for the study of games, and how blockchains may shape the future of game design and play.

Cryptogames, such as CryptoKitties, exemplify the phenomenon of blockchain-based gaming which has its own rules and mechanics of play. Our objective in this article has been first to demonstrate how blockchain-proven ownership of scarce tokens, virtual cats, is associated with their value in CryptoKitties, and second, how the breeding and marketplace aspects of the game function in terms of sustaining the game economy. This multilevel analysis has enabled a grounded discussion on the opportunities and business potential of blockchain-based game design, and also let us discover multiple weaknesses in it.

The idea of cryptogames as value-building mechanisms corresponds to the idea of the rules of a game that people play (Ganßmann, 2002), inspired by Wittgenstein's formulation of the definition of a game and applied to Bitcoin economies (Hütten \& Thiemann, 2018). As we have seen in this article, digital scarcity and the algorithmically limited supply of cryptocurrencies also reflect a special kind of digital 'materiality' that is the result of a semiotic process of the value construction (Maurer et al., 2013). Therefore, on one hand, the value of a kitty can be analysed as a semiotic construct, based on the meaningful distinctions between different traits and their scarcity; on the other hand, cryptocurrencies in use appear to be something different from the numeric representation of certain valuations, even if we are only talking about a game in this context. Cryptogames are a convenient tool to spot these differences.

Our conclusion is that there are three major factors determining the value of cryptogame tokens. First, blockchain as a socio-technical infrastructure sets the limits to what can be achieved within 
the network. Blockchains are hardly scalable. Operating a crypto wallet requires dedication and skill, and is not universally accessible. Second, the perceived materiality of tokens explains why smooth and frictionless transactions are currently not possible in cryptogames. That is because the maintenance of the infrastructure is paid by the users via transfers ('gas') to cryptocurrency miners. In such an unequal economic system, wealthier players can afford to make their move first, and players less eager to pay for transaction costs have to endure lag and standstill. Third, pseudo true ownership as a byproduct of the organization of the blockchain system makes it possible for players to enjoy the idea of anonymous ownership of game tokens even if their ownership is not legally validated outside of the game. The principle of anonymity and delegation of trust to algorithms and open data undermines any possibility of legal protection of full ownership. Therefore account recovery on a blockchain only becomes possible at the price of de-anonymization.

On top of these factors through which it is possible to understand the "gameness" of the cryptogame, we have suggested that in addition to (or replacing?) scarcity, there is digital abundance of game tokens which has led to their valuelessness. Our results indicate that these issues are connected the main challenges for designing viable gameplay in cryptogames: First, the limitations of blockchain technology, such as scalability, maintenance, anonymity, and trustlessness issues should be taken into account when designing an economic model based on it. Second, it is clear that scarcity has not worked as a grounding principle for such a model; instead, cryptogames have turned into economies of abundance and valuelessness. In fact, the notion of scarcity should be defined relationally, not absolutely. These challenges merit further studies, and on the basis of those, it will undoubtedly be possible to build better economic models for future cryptogames.

\section{References}

(all links checked on October 30, 2019)

Baldwin, J. (2018). In digital we trust: Bitcoin discourse, digital currencies, and decentralized network fetishism. Palgrave Communications 4(14). Retrieved from https://www.nature.com/articles/s41599-018-0065-0

Baudrillard, J. (1996). Global Debt and Parallel Universe. CTheory e031, Oct 16. [Transl. from the French publication in Libération, Paris, 1969.] Retrieved October 15, 2019, from http://ctheory.net/ctheory_wp/global-debt-and-parallel-universe/

Beck, R., Avital, M., Rossi, M. \& Thatcher, J. B. (2017). Blockchain Technology in Business and Information Systems Research. Business \& Information Systems Engineering 59(6), 381384. https://doi.org/10.1007/s12599-017-0505-1 
Blizzard End User License Agreement (2018). Retrieved October 15, 2019, from

https://www.blizzard.com/en-us/legal/2c72a35c-bflb-4ae6-99ec-80624e1b429c/blizzardend-user-license-agreement

Bogatyy, I. (2018, April 10). How we made \$100K trading CryptoKitties. Hackernoon. Retrieved from https://hackernoon.com/how-we-made-100k-trading-cryptokitties-2d69aebe715b cakepie99999 (2017, December 8). GETTING A CRYPTO KITTY!! [Video file] Retrieved from https://youtu.be/C9OeDd6hcOo

Calvão, F. (2019). Crypto-miners: Digital labor and the power of blockchain technology. Economic Anthropology 6(1), 123-134.

https://anthrosource.onlinelibrary.wiley.com/doi/pdf/10.1002/sea2.12136

Campbell-Verduyn, M. (2018). What are Blockchains and why are they relevant to Governance in the Global Political Economy. In M. Campbell-Verduyn (Ed.), Bitcoin and Beyond: Cryptocurrencies, Blockchains and Global Governance, 69-87. New York, NY: Routledge. Campbell-Verduyn, M., \& Goguen, M. (2018). The Mutual Constitution of Technology and Global Governance: Bitcoin, Blockchains, and the International Anti-Money Laundering Regime. In M. Campbell-Verduyn (Ed.), Bitcoin and Beyond: Cryptocurrencies, Blockchains and Global Governance, 69-87. New York, NY: Routledge.

Castronova, E. (2007). Exodus to the Virtual World. New York: St. Martin's Griffin.

Cheng, E. (2017, December 6). Meet CryptoKitties, the $\$ 100,000$ digital beanie babies epitomizing the cryptocurrency mania. $C N B C$. Retrieved from https://www.cnbc.com/2017/12/06/meetcryptokitties-the-new-digital-beanie-babies-selling-for-100k.html

Chohan, U.W. (2017). The Leisures of Blockchains: Exploratory Analysis. SSRN, Dec 4, 2017. http://dx.doi.org/10.2139/ssrn.3084411

CoinMarketCap, 2019. Retrieved from https://coinmarketcap.com/

CryptoKitties (2017) [Video game]. Developed by Axiom Zen \& Dapper Labs. Released on November 28, 2017. Retrieved from https://www.cryptokitties.co/

CryptoKitties (2018a, September 18). A New Category Is Here: Purrstige Traits! [Blog post]. Retrieved from https://medium.com/cryptokitties/a-new-trait-category-is-coming-f7ef3b3e4b85

CryptoKitties (2018b, March 22). Breeding fees demystified [Blog post]. Retrieved from https://medium.com/cryptokitties/why-love-isnt-free-for-cryptokitties-7cc00dd2bf6d 
CryptoKitties (2019, May 11) Bring your CryptoKitties to Dapper. [Blog post]. Retrieved from https://www.cryptokitties.co/blog/post/bring-your-cryptokitties-to-dapper

CryptoKitties (2018c, October 29). Some of the rarest CryptoKitties will stop being released on November 30th. [Blog post]. Retrieved from https://medium.com/cryptokitties/some-of-therarest-cryptokitties-will-stop-being-released-on-november-30th-ec $218 \mathrm{f} 3 \mathrm{fc} 5 \mathrm{c} 4$

CryptoKitties (2018d, November 16). What can I do with my CryptoKitty? [Blog post] Retrieved from https://www.cryptokitties.co/blog/post/when-you-purchase-a-cryptokitty-you-get-boththe-kitty-and-its-art

CryptoKitties Guide (2019). Retrieved from https://guide.cryptokitties.co/guide/cat-features/genes

CryptoKitties White Pa-Purr. CryptoKitties: Collectible and Breedable Cats Empowered by Blockchain Technology (2018). [Version 2.0.] Retrieved from https://drive.google.com/file/d/1soo-eAaJHzhw_XhFGMJp3VNcQoM43byS/view

CryptoKitties Sales. (2019). Retrieved from https://kittysales.herokuapp.com/

DappRadar (2019). Retrieved from https://dappradar.com

ETH Gas Station (2019). Retrieved from https://ethgasstation.info/

Etherscan.io (2019). Retrieved from https://etherscan.io/chart/etherprice

European Union. 2018. Directive (EU) 2018/843 of the European Parliament and of the Council of 30 May 2018, amending Directive (EU) 2015/849 on the prevention of the use of the financial system for the purposes of money laundering or terrorist financing, and amending Directives 2009/138/EC and 2013/36/EU. Retrieved from https://eurlex.europa.eu/eli/dir/2018/843/oj

Ferguson, R. (2019, February 11). Asset Interoperability: A Game Changer. [Blog post] The Gods Unchained blog. Retrieved from https://blog.godsunchained.com/2019/02/11/assetinteroperability-a-game-changer/

Ganßmann, H. (1988). Money - a symbolically generalized medium of communication? On the concept of money in recent sociology. Economy and Society 17(3), 285-316.

Garcia, D., Tessone, C. J., Mavrodiev, P. \& Perony, N. (2014). The digital traces of bubbles: feedback cycles between socio-economic signals in the Bitcoin economy. Journal of the Royal Society Interface 11(99). https://doi.org/10.1098/rsif.2014.0623

Gillespie, T. (2010). The politics of 'platforms'. New Media \& Society 12(3), 347-364.

Henrie, J. (2018, March 12). All about Family Jewels. [Blog post] Retrieved from https://medium.com/cryptokitties/all-about-family-jewels-ee4bc1f56608 
Hütten, M. \& Thiemann, M. (2018). Moneys at the margins - from political experiment to cashless societies. Bitcoin and Beyond: The Challenges and Opportunities of Blockchains for Global Governance. Malcolm Campbell-Verduyn (Ed.) New York \& London: Routledge, 25-47.

Kavanagh, D., Miscione, G. \& Ennis, P. J. (2019). The Bitcoin game: Ethno-resonance as method. Organization, Feb. 15, 2019. https://doi.org/10.1177/1350508419828567

Kitty Explorer (2019). Retrieved from https://www.kittyexplorer.com/

KittyHelper (2019). Retrieved from https://kittyhelper.co

Lamison-White, E. (2019, September 8). Everything wrong with Ethereum in 2019. Hackernoon. Retrieved from https://hackernoon.com/everything-wrong-with-ethereum-in-2019$\underline{\mathrm{r} 63 \mathrm{gl} 3 \mathrm{wr} 8}$

Lastowka, F. G. \& Hunter, D. (2004). The Laws of the Virtual Worlds. California Law Review, 92(1), 1-73.

Lehdonvirta, V. \& Castronova, E. (2014). Virtual Economies: Design and Analysis. Cambridge, MA: MIT Press.

Liu, J. (2017, December 12). The CryptoKitties Economy. Cryptocurrency Hub. Retrieved from https://cryptocurrencyhub.io/the-cryptokitties-economy-83c18ca07cd8

Madore, P.H. (2018, October 26) Chinese Civil Court Rules Bitcoin as Legally Protected Property. $C C N$. Retrieved from https://www.ccn.com/chinese-civil-court-bitcoin-are-legallyprotected-property

Mala, E. (2018, May 18). Who Spends \$140,000 on a CryptoKitty? The New York Times. Retrieved from https://www.nytimes.com/2018/05/18/style/cryptokitty-auction.html

Maurer, B., Nelms T. \& Swartz L. (2013). “When Perhaps the Real Problem Is Money Itself!” The Practical Materiality of Bitcoin. Social Semiotics 23(2), 261-277.

MEW Help Center (2018). MyEtherWallet Transactions: What is Gas? Retrieved from https://kb.myetherwallet.com/posts/transactions/what-is-gas/

Nakamoto, S. (2008). Bitcoin: A Peer-to-Peer Electronic Cash System [White Paper]. Retrieved from https://bitcoin.org/bitcoin.pdf

Nathaniell (2018, August 28). Can You Make Money With CryptoKitties? [Blog post] https://onemorecupof-coffee.com/can-you-make-money-with-cryptokitties/

Packard, A. (2010). Digital Media Law. Chichester: Wiley-Blackwell. 
Paez, D. (2018). The Bitcoin Bubble May Have Popped, but Blockchain Games Are Here to Stay. Inverse, Nov. 1, 2018. https://www.inverse.com/article/50424-cryptokitties-15-investmentblockchain-gaming?refresh $=46$

Parsons, T. (1963). On the Concept of Political Power. Proceedings of the American Philosophical Society 107(3), 232-262. https://www.jstor.org/stable/985582

Penney, J. W. (2012). Virtual Inequality: Challenges for the Net's Lost Founding Value. Northwestern Journal of Technology and Intellectual Property 10(3), 209-238. Retrieved from

https://scholarlycommons.law.northwestern.edu/cgi/viewcontent.cgi?article=1162\&context $=$ njtip

Ponomarev, E. (2019, January 29). DApp Survey Results 2019. State of DApps Ecosystem, Technology and Adoption [Blog post]. Retrieved from https://medium.com/fluencenetwork/dapp-survey-results-2019-a04373db6452

Pranked (2018). CryptoKitties Investing Essentials. Kitty.FYI. Retrieved October 15, 2019, from https://www.kitty.fyi/articles/cryptokitties-investing-essentials

Putney, D. (2019, January 2019). Cryptocollectible Collaboration: CryptoKitties And Gods Unchained Create Crossover Items. Ethnews.com. Retrieved from https://www.ethnews.com/cryptocollectible-collaboration-cryptokitties-and-gods-unchainedcreate-crossover-items

Scholten, O. J., Hughes, N. G. J., Deterding, S., Drachen, A., Walker, J. A., Zendle, D. I. (2019). Ethereum Crypto-Games: Mechanics, Prevalence and Gambling Similarities. CHI Play 2019.

Schroeder, S. (2017, December 4). How to play CryptoKitties, the insanely popular crypto game. Mashable. Retrieved from https://mashable.com/2017/12/04/how-to-play-cryptokitties/

Середа, Алеся. Цена и ценность криптовалютных токенов в игре CryptoKitties: магистерская работа / науч. рук. Орлова Г. А.; Европейский гуманитарный университет. - Вильнюс, 2019. [Serada, Alesja. 2019. Price and Value of Cryptocurrency Tokens in the Game CryptoKitties. Master's Thesis (in Russian). European Humanities University, Vilnius.]

Tomko, V. (2018). State of the Cryptogame Market. Crypto Games Conference, Nov. 13, 2018. Retrieved from https://www.youtube.com/watch?v=rajRT3vy-wA

Tomko, V. (2019). Cryptogame Market Right Now. Crypto Games Conference, Jul. 23, 2019. Retrieved from https://www.youtube.com/watch?v=h1G4st2vFzk 
Varshney, N. (2018, May 9). Someone paid \$170,000 for the most expensive CryptoKitty ever. TheNextWeb. Retrieved from https://thenextweb.com/hardfork/2018/09/05/most-expensivecryptokitty

Wood, K. \& Lindman, T. (2018, August 21). Why the next CryptoKitties mania won't be about collectables. TechCrunch. Retrieved from https://techcrunch.com/2018/08/21/why-the-nextcryptokitties-mania-wont-be-about-collectables/

Yli-Huumo J., Ko D., Choi S., Park S. \& Smolander K. (2016). Where Is Current Research on Blockchain Technology? - A Systematic Review. PLoS ONE 11(10): e0163477. https://doi.org/10.1371/journal.pone.0163477 